PROCEEDINGS OF THE

AMERICAN MATHEMATICAL SOCIETY

Volume 128, Number 3, Pages 735-743

$\mathrm{S}$ 0002-9939(99)05096-0

Article electronically published on July 8, 1999

\title{
FONCTIONS QUI OPERENT SUR LES ESPACES DE BESOV
}

\author{
DJALIL KATEB
}

(Communicated by Christopher D. Sogge)

\begin{abstract}
Soient $s, p$ et $q$ trois réels tels que $1<p<\infty, 1<s<1+1 / p$, et $1 \leq q \leq \infty$ et soit $f$ une fonction appartenant à l'espace de Besov $B_{p, q}^{s}\left(\mathbb{R}^{n}\right)$. Nous montrons que si $F$ est une fonction, de la variable réelle, nulle à l'origine, lipschitzienne et appartenant à l'espace $\dot{B}_{p, \infty}^{1+1 / p}$ on a alors $F(f) \in B_{p, q}^{s}\left(\mathbb{R}^{n}\right)$. La preuve est essentiellement basée sur des résultats d'approximation par des fonctions splines de degré 1 .
\end{abstract}

\section{INTRODUCTION}

On se propose de poursuivre l' exploration du calcul fonctionnel dans l' espace de Besov $B_{p, q}^{s}\left(\mathbb{R}^{n}\right)$. Il s' agit de décrire la classe des fonctions $F$, de la variable réelle, telle que $F(f) \in B_{p, q}^{s}\left(\mathbb{R}^{n}\right)$, pour tout $f \in B_{p, q}^{s}\left(\mathbb{R}^{n}\right)$ où $s, p$ et $q$ sont trois réels tels que $1<p<\infty, 1<s<1+1 / p$, et $1 \leq q \leq \infty$. Ce travail se situe dans une suite logique à celui accompli par Y.Meyer et G.Bourdaud [5] et indépendemment par P.Oswald [7] qui montrent que la fonction

$$
F(t)=|t|
$$

opère, dans ce cas précis, sur $B_{p, q}^{s}\left(\mathbb{R}^{n}\right)$. Il s'ensuit alors que deux classes $U$ et $V$ de fonctions "raisonnables" opèrent aussi: $U$ est l'ensemble des fonctions $F$ telles que

$$
F(0)=0, F^{\prime} \in L^{\infty}, \sup _{t \neq 0}\left|t \| F^{\prime \prime}(t)\right|<\infty
$$

tandis que $V$ est l'ensemble des fonctions $F$ telles que $F(0)=0$ et que $F^{\prime \prime}$ est une mesure bornée. Dans un travail en collaboration avec G.Bourdaud [4], nous décidions d'aller chercher des fonctions $F$ lipschitziennes, vérifiant $F(0)=0$, dans un espace de Besov homogène tel que $\dot{B}_{p, \infty}^{1+1 / p}$; il nous fallut cependant nous contenter d'un "pseudo" espace de Besov inclus dans $\dot{B}_{p, \infty}^{1+1 / p}$ mais suffisamment grand pour contenir les espaces $U$ et $V$.

Dans ce travail, on se propose d' améliorer ce résultat pour montrer que l'espace de Besov homogène $\dot{B}_{p, \infty}^{1+1 / p}$ est un bon candidat. Plus précisément, nous montrons le résultat suivant

Théorème. Soient $s, p$ et $q$ trois réels tels que $1<p<\infty, 1<s<1+1 / p$ et

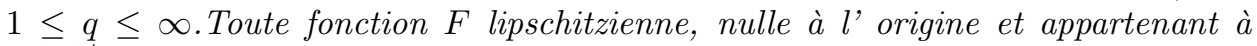
$\dot{B}_{p, \infty}^{1+1 / p}$ envoie par composition à gauche $B_{p, q}^{s}\left(\mathbb{R}^{n}\right)$ dans $B_{p, q}^{s}\left(\mathbb{R}^{n}\right)$. De plus, il

Received by the editors April 17, 1998.

1991 Mathematics Subject Classification. Primary 46E35, 47H30.

(C)1999 American Mathematical Society 
existe une constante $C>0$ (dépendant de $n, s, p$ et de q) telle que pour toute fonction $f \in B_{p, q}^{s}\left(\mathbb{R}^{n}\right)$, à valeurs réelles, on ait

$$
\|F(f)\|_{B_{p, q}^{s}\left(\mathbb{R}^{n}\right)} \leq C \operatorname{Max}\left(\left\|F^{\prime}\right\|_{\infty},\|F\|_{\dot{B}_{p, \infty}^{1+1 / p}}\right)\|f\|_{B_{p, q}^{s}\left(\mathbb{R}^{n}\right)} .
$$

\section{Espaces DE Besov}

Soient $1 \leq p \leq \infty, 1 \leq q \leq \infty$ et $0<s<1$. On définit l' espace de Besov $B_{p, q}^{s}=B_{p, q}^{s}\left(\mathbb{R}^{n}\right)$ comme suit:

Définition 1.1. Une fonction $f$ appartient à $B_{p, q}^{s}$ si et seulement si $f \in L^{p}\left(\mathbb{R}^{n}\right)$ et si:

$$
\omega(h)=\|f(x+h)-f(x)\|_{p}
$$

le module de continuité de $f$ en norme $L^{p}$ satisfait:

$$
\left(\int_{\mathbb{R}^{n}} \frac{\omega^{q}(h)}{|h|^{n+s q}} d h\right)^{1 / q}<\infty
$$

(avec la modification classique quand $p=\infty$ et/ou $q=\infty$ ). Si $s>1$, et $s \notin \mathbb{N}$ on écrit $s=m+r$ avec $m \in \mathbb{N}$ et $0<r<1$. Alors $f \in B_{p, q}^{s}$ si pour tout $\alpha \in \mathbb{N}^{n}$ tel que $|\alpha| \leq m$ on ait $\partial^{\alpha} f \in B_{p, q}^{r}$.

On note $\dot{B}_{p, q}^{s}$ l'espace homogène défini par

$$
f \in \dot{B}_{p, q}^{s} \Leftrightarrow\left(\int_{\mathbb{R}^{n}} \frac{\omega^{q}(h)}{|h|^{n+s q}} d h\right)^{1 / q}<\infty
$$

\section{Espaces de Besov et fonctions splines}

Placons nous dans le cas unidimensionnel $n=1$. Nous allons décrire une suite de résultats essentiels pour la preuve de notre théorème.

Soient $s, p, q$ trois réels tels que $1 \leq p<\infty, 1 \leq q \leq \infty$ et $1<s<1+1 / p$. Etant donnée une fonction $f$ appartenant à $B_{p, q}^{s}(\mathbb{R})$ nous notons par $f_{j}$ le spline de degré 1 ayant pour noeuds les points $x_{k}^{(j)}=2^{-j} k, k \in \mathbb{Z}$ et coincidant avec $f$ en ces points ( ceci a un sens car $f$ est continue ). On sait que si l' on note $\varphi=\chi * \chi, \chi$ étant la fonction caractéristique de l' intervalle $(0,1)$, il existe des constantes $c_{j k}$ telles que

$$
f_{j}(x)=\sum_{k} c_{j k} \varphi\left(2^{j} x-k\right)
$$

L' espace $B_{p, q}^{s}$ se caractérise d'une manière équivalente à l'aide des fonctions splines comme le montre le résultat suivant la

Proposition 2.1 ([4]). Si $1 \leq p<\infty, 1<s<1+1 / p$, on a alors

$$
\|f\|_{B_{p, p}^{s}} \approx\|f\|_{p}+\left\|\left(2^{j s}\left\|f-f_{j}\right\|_{p}\right)_{j \geq 0}\right\|_{l^{p}}
$$

Nous utiliserons, par la suite, aussi un résultat fort utile d'Oswald [7] on pose $\Delta f\left(k 2^{-j}\right)=f\left((k+1) 2^{-j}\right)-f\left(k 2^{-j}\right)$. On considère un sous-ensemble qu' on note $\Lambda$ (au lieu de $\Lambda^{j}$ ) du type $\Lambda=\{1,2, \ldots, m\} \subset \mathbb{N}$ ou $\Lambda=\mathbb{Z}$ et la série $\sum_{p_{\in} \Lambda}\left|\Delta f\left(k_{p} 2^{-j}\right)\right|^{p}$ dont les termes ont la propriété

$$
\Delta f\left(k_{p} 2^{-j}\right) . \Delta f\left(k_{p+1} 2^{-j}\right) \leq 0 .
$$

Précisons que les entiers $k_{p}$ sont tels que $k_{p}<k_{p+1}$. Oswald montre le 
Lemme 2.1 ([7]). Soient $p, q$, $s$ des réels tels que $1 \leq p<\infty, 1<s<1+1 / p$ et $1 \leq$ $q \leq \infty$. Il existe une constante $C>0$ telle que pour toute fonction $f$ appartenant $\grave{a} B_{p, q}^{s}$ et pour toute suite d' entiers $\left(k_{p}\right)_{p \in \Lambda}$ vérifiant $\Delta f\left(k_{p} 2^{-j}\right) \cdot \Delta f\left(k_{p+1} 2^{-j}\right) \leq 0$ on ait

$$
\left\|\left(2^{j(s-1 / p)} \cdot\left(\sum_{p \in \Lambda}\left|\Delta f\left(k_{p} 2^{-j}\right)\right|^{p}\right)^{1 / p}\right)_{j \geq 0}\right\|_{l^{q}} \leq C\|f\|_{\dot{B}_{p, q}^{s}} .
$$

La signification du lemme d'Oswald est la suivante: (2.1) montre que l'on a

$$
\|f\|_{B_{p, p}^{s}} \approx\|f\|_{p}+\left\|\left(2^{j(s-1 / p)} \cdot\left(\sum_{k}\left|\Delta^{2} f\left(k 2^{-j}\right)\right|^{p}\right)^{1 / p}\right)_{j \geq 0}\right\|_{l q},
$$

où $\Delta^{2}$ est la différence divisée seconde. Le lemme d'Oswald est puissant dans la mesure où il nous autorise à remplacer la différence seconde par une différence divisée première évaluée en des points dyadiques $\left(k_{p} 2^{-j}\right)$ tels que la propriété "d'oscillations" $\Delta f\left(k_{p} 2^{-j}\right) \Delta f\left(k_{p+1} 2^{-j}\right) \leq 0$ soit vérifiée.

Lemmes préparatoires. Signalons que la structure de ce travail est très voisine de celle présentée dans [4]. Nous présentons les deux lemmes essentiels directement liés à notre problème et l'amélioration - considérable- repose sur la preuve du second lemme.

Soit $F: \mathbb{R} \rightarrow \mathbb{R}$ une fonction lipschitzienne nulle en 0 appartenant à $\dot{B}_{p, \infty}^{1+1 / p}$. Nous désignerons par $t$ un réel tel que $1<s<t<1+1 / p$ et on posera $\sigma=t-1$. On a

Lemme 2.2. On a

i)

$$
\begin{gathered}
\int\left|F^{\prime}\left(f_{j+1}(x)\right)\right|^{p} \int \frac{\left|f_{j+1}^{\prime}(x)-f_{j}^{\prime}(x)-\left(f_{j+1}^{\prime}(y)-f_{j}^{\prime}(y)\right)\right|^{p}}{|x-y|^{p \sigma+1}} d y d x \\
\leq\left\|F^{\prime}\right\|_{\infty}^{p} \varepsilon_{j}^{p} 2^{p j(t-s)}
\end{gathered}
$$

où $\left(\varepsilon_{j}\right)$ est une suite appartenant à $l^{p}(\mathbb{N})$ telle que $\sum_{j \geq 0} \varepsilon_{j}^{p} \approx\|f\|_{B_{p, p}^{s}}^{p}$.

ii)

$$
\begin{aligned}
& \int\left|f_{j+1}^{\prime}(x)-f_{j}^{\prime}(x)\right|^{p} \int \frac{\left|F^{\prime}\left(f_{j+1}(x)\right)-F^{\prime}\left(f_{j+1}(y)\right)\right|^{p}}{|x-y|^{p \sigma+1}} d y d x \\
& \quad \leq \operatorname{Max}\left(\|F\|_{\dot{B}_{p, \infty}^{1+1 / p}}^{p},\left\|F^{\prime}\right\|_{\infty}^{p}\right) \varepsilon_{j}^{p} 2^{j p(t-s)}
\end{aligned}
$$

où $\left(\varepsilon_{j}\right)$ est une suite appartenant à $l^{p}(\mathbb{N})$ telle que $\sum_{j \geq 0} \varepsilon_{j}^{p} \approx\|f\|_{B_{p, p}^{s}}^{p}$.

Preuve. (Cf. [4].)

L'amélioration de notre résultat par rapport à celui exposé dans [4] réside dans le lemme suivant

Lemme 2.3. On $a$

$$
\begin{aligned}
& \int\left|f_{j}^{\prime}(x)\right|^{p}\left|F^{\prime}\left(f_{j+1}(x)\right)-F^{\prime}\left(f_{j}(x)\right)\right|^{p} d x \\
& \quad \leq C \operatorname{Max}\left(\left\|F^{\prime}\right\|_{\infty}^{p},\left(\|F\|_{\dot{B}_{p, \infty}^{1+1 / p}}\right)^{p}\right) \varepsilon_{j}^{p} 2^{j p(1-s)}
\end{aligned}
$$

où $\left(\varepsilon_{j}\right)$ est une suite appartenant à $l^{p}(\mathbb{N})$ telle que $\sum_{j \geq 0} \varepsilon_{j}^{p} \approx\|f\|_{B_{p, p}^{s}}^{p}$. 
Preuve. Considérons la partition suivante:

$$
\mathbb{R}=\bigcup_{m \in \Lambda} I_{m}
$$

où $\Lambda$ est du type $\{1,2, \ldots, m\} \subset \mathbb{N}$ ou $\Lambda=\mathbb{Z}$ et où les $I_{m}$ sont tels que si $f_{j}$ est croissante sur $I_{m}$ alors $f_{j}$ est strictement décroissante sur $I_{m+1}$. Soit $K_{m}$ l'ensemble des entiers $k$ tels que l' intervalle $\left(k 2^{-j},(k+1) 2^{-j}\right)$ soit inclus dans $I_{m}$. On a:

$$
\begin{gathered}
\int\left|f_{j}^{\prime}(x)\right|^{p}\left|F^{\prime}\left(f_{j+1}(x)\right)-F^{\prime}\left(f_{j}(x)\right)\right|^{p} d x \\
=\sum_{m \in \Lambda} \sum_{k \in K_{m}} \int_{k 2^{-j}}^{(k+1) 2^{-j}}\left|f_{j}^{\prime}(x)\right|^{p}\left|F^{\prime}\left(f_{j+1}(x)\right)-F^{\prime}\left(f_{j}(x)\right)\right|^{p} d x \\
\leq C\left(I_{1}+I_{2}\right)
\end{gathered}
$$

où on a posé

$$
I_{1}=\sum_{m \in \Lambda} \sum_{k \in K_{m}} \int_{k 2^{-j}}^{(k+1) 2^{-j}}\left|F^{\prime}\left(f_{j+1}(x)\right) f_{j+1}^{\prime}(x)-F^{\prime}\left(f_{j}(x)\right) f_{j}^{\prime}(x)\right|^{p} d x
$$

et

$$
I_{2}=\sum_{m \in \Lambda} \sum_{k \in K_{m}} \int_{k 2^{-j}}^{(k+1) 2^{-j}}\left|F^{\prime}\left(f_{j}(x)\right)\right|^{p}\left|f_{j+1}^{\prime}(x)-f_{j}^{\prime}(x)\right|^{p} d x .
$$

Seule la majoration de $I_{1}$ nous intéresse vu que celle de $I_{2}$, conséquence immédiate du lemme 2.1, est donnée par

$$
I_{2} \leq C\left\|F^{\prime}\right\|_{\infty}^{p} \varepsilon_{j}^{p} 2^{j p(1-s)}
$$

où $\left(\varepsilon_{j}\right)$ est une suite appartenant à $l^{p}(\mathbb{N})$ telle que $\sum_{j \geq 0} \varepsilon_{j}^{p} \approx\|f\|_{B_{p}^{s}, p}^{p}$.

Majoration de $I_{1}$. Si

$$
g(x)=F^{\prime}\left(f_{j+1}(x)\right) f_{j+1}^{\prime}(x)-F^{\prime}\left(f_{j}(x)\right) f_{j}^{\prime}(x),
$$

on a $\int_{k 2^{-j}}^{(k+1) 2^{-j}} g(x) d x=0$. Notons $\Omega_{j h}^{k}=\left\{x \in\left(k 2^{-j},(k+1) 2^{-j}\right) / x+h \in\right.$ $\left.\left(k 2^{-j},(k+1) 2^{-j}\right)\right\},|h| \leq 2^{-j}$, on a d'après l'inégalité de Holder

$$
\int_{k 2^{-j}}^{(k+1) 2^{-j}}|g(x)|^{p} d x \leq 2^{j} \int_{|h| \leq 2^{-j}} \int_{x \in \Omega_{j h}^{k}}|g(x+h)-g(x)|^{p} d x d h .
$$

On a ainsi $I_{1} \leq C\left(I_{3}+I_{4}\right)$ où on a posé

$$
I_{3}=\sum_{m \in \Lambda} \sum_{k \in K_{m}} \sup _{|h| \leq 2^{-j}} \int_{x \in \Omega_{j h}^{k}}\left|F^{\prime}\left(f_{j}(x+h)\right) f_{j}^{\prime}(x+h)-F^{\prime}\left(f_{j}(x)\right) f_{j}^{\prime}(x)\right|^{p} d x
$$

et où

$$
\begin{aligned}
I_{4}=\sum_{m \in \Lambda} \sum_{k \in K_{m}} 2^{j} \int_{|h| \leq 2^{-j}} \int_{x \in \Omega_{j h}^{k}} \mid F^{\prime}\left(f_{j+1}(x+h)\right) f_{j+1}^{\prime}(x+h) & \\
& -\left.F^{\prime}\left(f_{j+1}(x)\right) f_{j+1}^{\prime}(x)\right|^{p} d x .
\end{aligned}
$$


On commence par majorer $I_{3}$. L' estimation de $I_{3}$ est assez simple. En effet, si on note $J_{k}$ l'image de $\left(k 2^{-j},(k+1) 2^{-j}\right)$ par $f_{j}$, on a via un changement de variables:

$$
I_{3} \leq C \sum_{m \in \Lambda} \sum_{k \in K_{m}} 2^{j(p-1)}\left|\Delta f\left(k 2^{-j}\right)\right|^{p-1} \sup _{h} \int_{x \in J_{k}}\left|F^{\prime}(x+h)-F^{\prime}(x)\right|^{p} d x
$$

(le sup étant pris pour $|h| \leq\left|f\left(k 2^{-j}\right)-f\left((k+1) 2^{-j}\right)\right|$ ). Les intervalles $J_{k}$ étant deux à deux disjoints, on a alors

$$
I_{3} \leq C 2^{j p} \sum_{m \in \Lambda} 2^{-j}\left|\Delta f\left(l_{m} 2^{-j}\right)\right|^{p}\|F\|_{\dot{B}_{p, \infty}^{1+1 / p}}^{p}
$$

où $l_{m}$ est l'indice tel que $\left|\Delta f\left(l_{m} 2^{-j}\right)=\sup _{k \in K_{m}}\right| \Delta f\left(k 2^{-j}\right) \mid$. Le lemme d'Oswald nous permet alors de conclure que

$$
I_{3} \leq\|F\|_{\dot{B}_{p, \infty}^{1+1 / p}}^{p} \varepsilon_{j}^{p} 2^{j p(1-s)}
$$

où $\left(\varepsilon_{j}\right)$ est une suite appartenant à $l^{p}(\mathbb{N})$ telle que $\sum_{j \geq 0} \varepsilon_{j}^{p} \approx\|f\|_{B_{p, p}^{s}}^{p}$.

La clé de la preuve du lemme repose essentiellement sur l'estimation assez compliquée de $I_{4}$. Pour $|h| \leq 2^{-j-1}$, on note

$$
\begin{gathered}
\left.\Omega_{h, k}^{(1)}=\left\{x \in\left(\left(k 2^{-j},(k+1 / 2) 2^{-j}\right)\right) / x+h \in\left(k 2^{-j},(k+1 / 2) 2^{-j}\right)\right)\right\}, \\
\Omega_{h, k}^{(2)}=\left\{x \in\left((k+1 / 2) 2^{-j},(k+1) 2^{-j}\right) / x+h \in\left((k+1 / 2) 2^{-j},(k+1) 2^{-j}\right)\right\}, \\
\Omega_{h, k}^{(3)}=\left\{x \in\left(k 2^{-j},(k+1 / 2) 2^{-j}\right) / x+h \in\left((k+1 / 2) 2^{-j},(k+1) 2^{-j}\right)\right\}, \\
\left.\Omega_{h, k}^{(4)}=\left\{x \in\left((k+1 / 2) 2^{-j},(k+1) 2^{-j}\right) / x+h \in\left(k 2^{j},(k+1 / 2) 2^{-j}\right)\right)\right\} .
\end{gathered}
$$

On décompose $I_{4} \leq C\left(I_{4}^{(1)}+I_{4}^{(2)}+I_{4}^{(3)}+I_{4}^{(4)}\right)$ où

$$
\begin{aligned}
I_{4}^{(1)}=\sum_{m \in \Lambda} \sum_{k \in K_{m}} \sup _{|h| \leq 2^{-j-1}} \int_{x \in \Omega_{h, k}^{(1)}} \mid F^{\prime}\left(f_{j+1}(x+h)\right) f_{j+1}^{\prime}(x+h) & -\left.F^{\prime}\left(f_{j+1}(x)\right) f_{j+1}^{\prime}(x)\right|^{p} d x,
\end{aligned}
$$

où

$$
\begin{aligned}
I_{4}^{(2)}=\sum_{m \in \Lambda} \sum_{k \in K_{m}} \sup _{|h| \leq 2^{-j-1}} \int_{x \in \Omega_{h, k}^{(2)}} \mid F^{\prime}\left(f_{j+1}(x+h)\right) f_{j+1}^{\prime}(x+h) & \\
& -\left.F^{\prime}\left(f_{j+1}(x)\right) f_{j+1}^{\prime}(x)\right|^{p} d x,
\end{aligned}
$$

où

$$
\begin{aligned}
I_{4}^{(3)}=\sum_{m \in \Lambda} \sum_{k \in K_{m}} 2^{j} \int_{|h| \leq 2^{-j-1}} \int_{x \in \Omega_{h, k}^{(3)}} \mid F^{\prime}\left(f_{j+1}(x+h)\right) f_{j+1}^{\prime}(x+h) & \\
& -\left.F^{\prime}\left(f_{j+1}(x)\right) f_{j+1}^{\prime}(x)\right|^{p} d x d h,
\end{aligned}
$$

et où

$$
\begin{array}{r}
I_{4}^{(4)}=\sum_{m \in \Lambda} \sum_{k \in K_{m}} 2^{j} \int_{|h| \leq 2^{-j-1}} \int_{x \in \Omega_{h, k}^{(4)}} \mid F^{\prime}\left(f_{j+1}(x+h)\right) f_{j+1}^{\prime}(x+h) \\
-\left.F^{\prime}\left(f_{j+1}(x)\right) f_{j+1}^{\prime}(x)\right|^{p} d x d h .
\end{array}
$$


Les majorations de $I_{4}^{(1)}$ et de $I_{4}^{(2)}$ sont directes. En effet, remarquons que l'on a

$$
\begin{aligned}
& \left|f\left(\left(k+\frac{1}{2}\right) 2^{-j}\right)-f\left(k 2^{-j}\right)\right| \\
& \quad \leq\left|f\left((k+1) 2^{-j}\right)-f\left(k 2^{-j}\right)\right|+\frac{1}{2}\left|f\left(k 2^{-j}\right)-2 f\left(\left(k+\frac{1}{2}\right) 2^{-j}\right)+f\left((k+1) 2^{-j}\right)\right|
\end{aligned}
$$

par changement de variable on obtient alors

$$
\begin{gathered}
I_{4}^{(1)} \leq C \operatorname{Max}\left(\left\|F^{\prime}\right\|_{\infty}^{p},\|F\|_{\dot{B}_{p, \infty}^{1+1 / p}}^{p}\right) \\
\left(\sum_{m \in \Lambda} 2^{j(p-1)}\left|\Delta f\left(2^{(-j)} l_{m}\right)\right|^{p}+2^{j(p-1)} \sum_{m \in \Lambda}\left|\Delta f\left(2^{-j} l_{m}\right)\right|^{p-1} \delta_{l_{m}^{\prime}}\right)
\end{gathered}
$$

où on a noté $\delta_{k}=\frac{1}{2}\left|f\left((k+1) 2^{-j}\right)-2 f\left(\left(k+\frac{1}{2}\right) 2^{-j}\right)+f\left(k 2^{-j}\right)\right|$ et où $\delta_{l_{m}^{\prime}}=$ $\sup _{k \in K_{m}} \delta_{k}$. En appliquant l'inégalité de Holder et le lemme d'Oswald aidant, on alors:

$$
I_{4}^{(1)} \leq C \operatorname{Max}\left(\left\|F^{\prime}\right\|_{\infty}^{p},\left(\|F\|_{\dot{B}_{p}^{1+\infty}}^{1+p}\right)^{p}\right) 2^{j p(1-s)} \varepsilon_{j}^{p} .
$$

En ce qui concerne $I_{4}^{(2)}$, on suit exactement la même démarche pour avoir

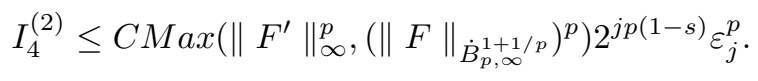

Reste à étudier de près $I_{4}^{(3)}$ et $I_{4}^{(4)}$. Tout d'abord, on s'intéressera aux intervalles $\left(k 2^{-j},(k+1 / 2) 2^{-j}\right), k \in K_{m}$, sur lesquels $f_{j+1}(x)$ a la même monotonie que $f_{j}(x)$; en effet dans le cas contraire, on a $\left|f_{j+1}^{\prime}\right| \leq\left|f_{j+1}^{\prime}-f_{j}^{\prime}\right|$.

On majore tout d'abord, quand $k \in K_{m}$ :

$$
2^{j} \int_{|h| \leq 2^{-j-1}} \int_{x \in \Omega_{h, k}^{(3)}}\left|F^{\prime}\left(f_{j+1}(x+h)\right)-F^{\prime}\left(f_{j+1}(x)\right)\right|^{p}\left|f_{j+1}^{\prime}(x)\right|^{p} d x d h .
$$

On peut toujours supposer, quitte à utiliser l'inégalité triangulaire, que l'on a $\left|f_{j+1}^{\prime}(x)\right| \leq\left|f_{j+1}^{\prime}(x+h)\right|$, quand $x \in \Omega_{h, k}^{(3)},|h| \leq 2^{-j-1}$. On a alors si on note $d_{j k}=f_{j+1}^{\prime}(x)$ quand $x \in\left(k 2^{j},(k+1 / 2) 2^{-j}\right)$

$$
\begin{gathered}
2^{j} \int_{|h| \leq 2^{-j-1}} \int_{x \in \Omega_{h, k}^{(3)}}\left|F^{\prime}\left(f_{j+1}(x+h)\right)-F^{\prime}\left(f_{j+1}(x)\right)\right|^{p}\left|f_{j+1}^{\prime}(x)\right|^{p} d x d h \\
\leq C 2^{j} d_{j k}^{p-2} \int_{|h| \leq 2^{-j-1}} \int_{x \in \Omega_{h, k}^{(3)}}\left|F^{\prime}\left(f_{j+1}(x+h)\right)-F^{\prime}\left(f_{j+1}(x)\right)\right|^{p} \\
\times\left|f_{j+1}^{\prime}(x)\right| f_{j+1}^{\prime}(x+h) d x d h .
\end{gathered}
$$

Notons $J_{k}^{(3)}$ l'image par $f_{j+1}$ de $\Omega_{h, k}^{(3)}$. Par un changement de variables on a alors

$$
\begin{gathered}
\int_{|h| \leq 2^{-j-1}} \int_{x \in \Omega_{h, k}^{(3)}}\left|F^{\prime}\left(f_{j+1}(x+h)\right)-F^{\prime}\left(f_{j+1}(x)\right)\right|^{p}\left|f_{j+1}^{\prime}(x)\right|^{p} d x d h \\
\leq C 2^{j(p-1)}\left|f\left((k+1 / 2) 2^{-j}\right)-f\left(k 2^{-j}\right)\right|^{p} \\
\times \sup _{|h| \leq\left|f\left((k+1 / 2) 2^{j}\right)-f\left(k 2^{-j}\right)\right|} \frac{1}{h} \int_{J_{k}^{(3)}}\left|F^{\prime}(u+h)-F^{\prime}(u)\right|^{p} d u .
\end{gathered}
$$


En utilisant le même argument que pour (2.9), on déduit alors grâceà la lemme 2.1 et l'inégalité triangulaire que l'on a

$$
\begin{gathered}
I_{4}^{(3)} \leq C \operatorname{Max}\left(\left\|F^{\prime}\right\|_{\infty}^{p},\left(\|F\|_{\left.\dot{B}_{p, \infty}^{1+1 / p}\right)^{p}}\right)\right. \\
\left(2^{-j} \sum_{m \in \Lambda} 2^{j(p-1)}\left|f\left(\left(k_{m}+1 / 2\right) 2^{-j}\right)-f\left(k_{m} 2^{-j}\right)\right|^{p}+2^{j p(1-s)} \epsilon_{j}^{p}\right)
\end{gathered}
$$

et le lemme d'Oswald permet alors de conclure que

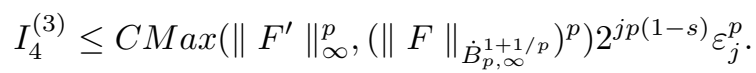

La majoration de $I_{4}^{(4)}$ est identique. En rassemblant l'ensemble des inégalités obtenues, on a alors la majoration désirée.

On déduit ainsi le résultat suivant

Corollaire 2.1. On a

$$
\begin{aligned}
& \iint \frac{\left|F^{\prime}\left(f_{j+1}(x)\right)\left[f_{j+1}^{\prime}(x)-f_{j}^{\prime}(x)\right]-F^{\prime}\left(f_{j+1}(y)\right)\left[f_{j+1}^{\prime}(y)-f_{j}^{\prime}(y)\right]\right|^{p}}{|x-y|^{p \sigma+1}} d y d x \\
& \leq \operatorname{Max}\left(\left\|F^{\prime}\right\|_{\infty}^{p},\|F\|_{\dot{B}_{p}^{1+\infty}}^{p} \varepsilon_{j}^{p+p}\right) \varepsilon_{j}^{p} 2^{j p(t-s)}
\end{aligned}
$$

où $\left(\varepsilon_{j}\right)$ est une suite appartenant à $l^{p}(\mathbb{N})$ telle que $\sum_{j \geq 0} \varepsilon_{j}^{p} \approx\|f\|_{B_{p, p}^{s}}^{p}$.

Nous avons aussi besoin du lemme suivant

Lemme 2.4 ([4]). On a

$$
\begin{gathered}
\iint \frac{\mid f_{j}^{\prime}(x)\left[F^{\prime}\left(f_{j+1}(x)\right)-F^{\prime}\left(f_{j}(x)\right)\right]-f_{j}^{\prime}(y)\left[F^{\prime}\left(f_{j+1}(y)\right)-\left.F^{\prime}\left(f_{j}(y)\right]\right|^{p}\right.}{|x-y|^{p \sigma+1}} d y d x \\
\leq \operatorname{Max}\left(\|F\|_{\dot{B}_{p, \infty}^{1+1 / p}}^{p}\left\|F^{\prime}\right\|_{\infty}^{p}\right) \varepsilon_{j}^{p} 2^{j p(t-s)}
\end{gathered}
$$

où $\left(\varepsilon_{j}\right)$ est une suite appartenant à $l^{p}(\mathbb{N})$ telle que $\sum_{j \geq 0} \varepsilon_{j}^{p} \approx\|f\|_{B_{p, p}^{s}}^{p}$.

Après cette préparation, nous sommes en mesure de prouver le théorème principal.

\section{Preuve du RÉsultat Principal}

Le fait que $\mathrm{F}$ soit lipschitzienne et nulle en 0 implique l'appartenance de $F(f)$ à $\mathbf{L}^{p}$. Plus précisément, on a

$$
\left\|F(f)-F\left(f_{j}\right)\right\|_{p} \leq\left\|F^{\prime}\right\|_{\infty} \varepsilon_{j} 2^{-j s} .
$$

Soit $t$ un réel tel que $s<t<1+1 / p$. Montrons qu'il existe une constante $C>0$ (dépendant de $s, t$ et de $p$ ) et une suite $\left(\varepsilon_{j}\right)_{j \geq 0}$ appartenant à $l^{p}$ telle que l' on ait

$$
\left\|F\left(f_{j}\right)\right\|_{\dot{B}_{p, p}^{t}} \leq C \operatorname{Max}\left(\left\|F^{\prime}\right\|_{\infty},\|F\|_{\dot{B}_{p, \infty}^{1+1 / p}}\right) 2^{j(t-s)} \varepsilon_{j} .
$$

Soit $g_{j}=F\left(f_{j+1}\right)-F\left(f_{j}\right)$; on a

$$
\left\|F\left(f_{j+1}\right)-F\left(f_{j}\right)\right\|_{\dot{B}_{p, p}^{t}}^{p} \leq C\left(A_{1}+A_{2}\right)
$$

où

$$
A_{1}=\iint \frac{\left|F^{\prime}\left(f_{j+1}(x)\right)\left[f_{j+1}^{\prime}(x)-f_{j}^{\prime}(x)\right]-F^{\prime}\left(f_{j+1}(y)\right)\left[f_{j+1}^{\prime}(y)-f_{j}^{\prime}(y)\right]\right|^{p}}{|x-y|^{p \sigma+1}} d y d x
$$


et

$$
A_{2}=\iint \frac{\mid f_{j}^{\prime}(x)\left[F^{\prime}\left(f_{j+1}(x)\right)-F^{\prime}\left(f_{j}(x)\right)\right]-f_{j}^{\prime}(y)\left[F^{\prime}\left(f_{j+1}(y)\right)-\left.F^{\prime}\left(f_{j}(y)\right]\right|^{p}\right.}{|x-y|^{p \sigma+1}} d y d x .
$$

Le corollaire 2.1 et le lemme 2.4 permettent de déduire que

$$
\left\|F\left(f_{j+1}\right)-F\left(f_{j}\right)\right\|_{\dot{B}_{p, p}^{t}} \leq \operatorname{Max}\left(\left\|F^{\prime}\right\|_{\infty},\|F\|_{\dot{B}_{p, \infty}^{1+1 / p}}\right) 2^{j(t-s)} \varepsilon_{j},
$$

tandis que l' inégalité triangulaire et (3.3) impliquent

$$
\left\|F\left(f_{j}\right)\right\|_{\dot{B}_{p, p}^{t}} \leq\left\|F\left(f_{0}\right)\right\|_{\dot{B}_{p, p}^{t}}+C \operatorname{Max}\left(\left\|F^{\prime}\right\|_{\infty},\|F\|_{\dot{B}_{p, \infty}^{1+1 / p}}\right)\left(\sum_{k=0}^{j-1} \varepsilon_{k} 2^{k(t-s)}\right) .
$$

L' injection $l^{1} * l^{p} \hookrightarrow l^{p}$, nous apprend que la suite $\left(\eta_{j}\right)_{j>0}$ définie par

$$
\eta_{j}=2^{-j(t-s)} \sum_{k=0}^{j-1} \varepsilon_{k} 2^{k(t-s)}
$$

appartient à $l^{p}$ avec l' estimation

$$
\left\|\left(\eta_{j}\right)_{j>0}\right\|_{l^{p}} \leq C\left\|\left(\varepsilon_{j}\right)_{j>0}\right\|_{l^{p}}
$$

et on en déduit ainsi qu'il existe une constante positive $C=C(s, p, t)$ telle que l' on ait

$$
\left\|F\left(f_{j}\right)\right\|_{\dot{B}_{p, p}^{t}} \leq C \operatorname{Max}\left(\left\|F^{\prime}\right\|_{\infty},\|F\|_{\dot{B}_{p, \infty}^{1+1 / p}}\right)\left(\left\|f_{0}\right\|_{p}+2^{j(t-s)} \eta_{j}\right) .
$$

Reste le terme en $f_{0}$. On a

$$
\begin{gathered}
\left\|f_{0}\right\|_{p} \leq\left\|f-f_{0}\right\|_{p}+\|f\|_{p} \leq c\|f\|_{B_{p, p}^{s}} \\
\leq c 2^{j(t-s)} 2^{-j(t-s)}\|f\|_{B_{p, p}^{s}} .
\end{gathered}
$$

En rassemblant les inégalités, on déduit alors qu' il existe une constante positive $C=C(s, t, p)$ telle que l' on ait

$\left\|F\left(f_{j}\right)\right\|_{\dot{B}_{p, p}^{t}} \leq C \operatorname{Max}\left(\left\|F^{\prime}\right\|_{\infty},\|F\|_{\dot{B}_{p}^{1+1}, \infty}\right)\left(2^{j(t-s)} 2^{-j(t-s)}\|f\|_{B_{p, p}^{s}+2^{j(t-s)}} \eta_{j}\right)$, grâce aux estimations (3.5) et $\sum_{j \geq 0} \varepsilon_{j}^{p} \leq C\|f\|_{B_{p, p}^{s}}^{p}$, on a alors

$$
\|F(f)\|_{B_{p, p}^{s}} \leq C \operatorname{Max}\left(\left\|F^{\prime}\right\|_{\infty},\|F\|_{\dot{B}_{p, \infty}^{1+1 / p}}\right)\|f\|_{B_{p, p}^{s}} .
$$

Ceci achève la preuve du théorème principal dans le cas $n=1$ et $p=q$.

La preuve du théorème principal dans le cas $n>1, p \neq q$ se fait en deux étapes:

1 ère étape: On étend le résultat à $B_{p, p}^{s}\left(\mathbb{R}^{n}\right)$ grâce à la propriété de Fubini. Ceci prouve alors le théorème principal quand $p=q$.

2ème étape: on étend au cas $B_{p, q}^{s}\left(\mathbb{R}^{n}\right)$ en suivant la démarche de BourdaudMeyer ([5], p 356-357 / [4], p673-675). 


\section{BiBLIOGRAPHIE}

1. Bourdaud, G., Fonctions qui opèrent sur les espaces de Sobolev, Sem. Anal. Harm. Orsay, (1980-81), 6-17. MR 84c:46031

2. Bourdaud, G., Kateb, Dalila, Fonctions qui opèrent sur les espaces de Besov, Proc. Amer. Math. Soc., 116 (1991), 1067-1076. MR 91j:46040

3. Bourdaud, G. Kateb, Djalil, Le calcul fonctionnel dans l' espace de Sobolev fractionnaire, Math.Z., 210 (1992), 607-613. MR 93i:46058

4. Bourdaud, G. Kateb, Djalil, Fonctions qui opèrent sur les espaces de Besov, Math.Ann, 330 (1995), 653-675. MR 96k:47118

5. Bourdaud, G. Meyer, Y., Fonctions qui opèrent sur les espaces de Sobolev, J. Functional Anal., 97 (1991), 351-360. MR 92e:46062

6. Dahlberg, B. J., A note on Sobolev spaces, Proc. Symp. Pure Math., 35, 1 (1979), 183-185. MR 81h:46030

7. Oswald, P., On the boundedness of the mapping $f \rightarrow|f|$ in Besov spaces, Comment. Univ. Carolinae, 33 (1992), 57-66. MR 93c:46052

Université de Technologie de Compiègne, Centre de Recherches de Royallieu, B. P. 20529, 60205 COMPIÈGne CEDEX, France

E-mail address: dkateb@dma.utc.fr 